\title{
The Surgical Apgar Score and frailty as outcome predictors in short- and long-term evaluation of fit and frail older patients undergoing elective laparoscopic cholecystectomy - a prospective cohort study
}

\author{
Kinga Mastalerz ${ }^{1}$, Jakub Kenig ${ }^{1}$, Urszula Olszewska ${ }^{1}$, Cyprian Michalik ${ }^{2}$ \\ ${ }^{1}$ Department of General, Oncologic and Geriatric Surgery, Jagiellonian University Medical College, Krakow, Poland \\ 2Department of Urology, Ludwik Rydygier Memorial Specialized Hospital, Krakow, Poland
}

Videosurgery Miniinv 2018; 13 (3): 350-357

DOI: https://doi.org/10.5114/wiitm.2018.75878

\begin{abstract}
Introduction: Frailty increases the risk of poor surgical outcomes in the older population. Some intraoperative factors may also influence the final result and can be evaluated. The Surgical Apgar Score (SAS) is a simple system predicting postoperative mortality and morbidity. However, the utility of the SAS remains unknown in fit and frail older patients undergoing elective laparoscopic cholecystectomy due to benign gallbladder diseases.

Aim: To evaluate the usefulness of the SAS in predicting 30-day morbidity and 1-year mortality in older fit and frail patients undergoing elective laparoscopic cholecystectomy.

Material and methods: Consecutive patients ( $\geq 70$ years) were enrolled in the prospective study. The Comprehensive Geriatric Assessment (CGA) was used to diagnose frailty. Logistic regression was conducted to investigate the association between the scores and the outcomes.

Results: The study included 144 consecutive older patients with a median age of 76 (range: 70-91) years. The prevalence of frailty was $44.4 \%$. The 30-day mortality and morbidity were $0 \%$ and $11.8 \%$, respectively. The 1-year mortality was $6.3 \%$ and 7 out of 9 occurred in the frail group. SAS $<7$ points was identified as an independent predictor of 30-day postoperative morbidity $(O R=5.1 ; 95 \% \mathrm{Cl}: 1.5-18.1)$. Age $>85$ years $(O R=1.9 ; 95 \% \mathrm{Cl}: 1.2-16.4)$ and frailty $(O R=3.4 ; 95 \% \mathrm{Cl}: 1.1-19.3)$ were predictors of 1-year mortality.

Conclusions: Laparoscopic cholecystectomy can be safely performed in older fit and frail patients. The SAS, not age, turned out to be the most important predictor of 30-day morbidity. Frailty and age > 85 years were predictors of 1-year mortality. Older patients with SAS $<7$ points should be followed meticulously in order to diagnose and treat potential complications early on.
\end{abstract}

Key words: laparoscopic cholecystectomy, elderly, frailty, Surgical Apgar Score, older patients.

\section{Introduction}

Elective laparoscopic cholecystectomy is a safe and effective treatment method in fit older patients, achieving outcomes comparable with their younger counterparts [1]. However, older patients are very heterogeneous with regard to physical reserve and co-morbidity. Particularly, this concerns frail older patients who have increased vulnerability and loss of adaptive capacity to external stressors (including surgery), which results in an increased risk of adverse outcomes [2]. In our previous study, we

\section{Address for correspondence}

Assoc. Prof. Jakub Kenig MD, PhD, $3^{\text {rd }}$ Department of General, Oncologic and Geriatric Surgery, Jagiellonian University Medical College, 35-37 Pradnicka St, 31-202 Krakow, Poland, phone: +48 12633 19 95, +48 500091 400, e-mail: +48 500 091 400, e-mail: jakub.kenig@uj.edu.pl 
demonstrated that emergency surgery in this group of older patients is associated with poor outcomes and an elective surgery in experienced hands could be a safe option for frail patients [3].

We hypothesize that the body's reaction to the surgical injury could be the key element in this group of patients. Thus, there is a great need to evaluate not only the preoperative health status, but also the surgical intervention itself. The Surgical Apgar Score (SAS), proposed by Gawande et al., is a simple and rapid scoring system, using only three elements extracted from anaesthesia records (estimated blood loss, lowest intraoperative heart rate and lowest intraoperative mean arterial pressure) [4]. It was validated in a large cohort of general surgical patients, and also by our team, in older patients undergoing emergency surgery $[5,6]$. However, it remains unknown whether the SAS might be useful in older fit and frail patients undergoing elective cholecystectomy, which is one of the most common surgical procedures in the older population.

\section{Aim}

Therefore, the aim of this study was to evaluate the usefulness of the SAS in predicting 30-day morbidity and 1-year mortality in older fit and frail patients undergoing elective laparoscopic cholecystectomy.

\section{Material and methods}

\section{Study population}

Between January 2013 and December 2016, consecutive patients, aged 70 years and older, who were in need of elective laparoscopic cholecystectomy were included in the prospective study. The Ethics Committee approved this study and informed consent was obtained from all patients or their caregivers. Patients who had open cholecystectomy or gallbladder cancer in the final pathological report were excluded.

\section{Study protocol}

All patients had the CGA, which was performed between 1 and 7 days before hospital admission (in most cases, on the day of admission) by trained physicians of the geriatric team. The CGA included instruments evaluating four cardinal domains of geriatric assessment:

- Functional status: activities of daily living (ADL; cut-off score < 5) [7] and Instrumental Activities of Daily Living (IADL; cut-off score $\leq 7$ ) [8].
- Psychological status: the Blessed OrientationMemory-Concentration (BOMC; cut-off score > 10) test [9], the Clock Drawing Test (CDT; cut-off score > 3) [10], the Geriatric Depression Scale (GDS) [11].

- Physical health: Comorbidities: the Charlson Comorbidity Index (CCS) [12], the full version of mini nutritional assessment (MNA; cut-off score < 24) [13], polypharmacy assessment (cut-off score $>7$ medications/day) [14], the Timed Up and Go (TUG) [15].

- Social factors: Medical Outcomes Study Social Support Scale (MOS-SSS; cut-off score < 4) [16].

Each test was scored on a dichotomous scale, based on whether there was or was not an impairment in any of the parameters.

Additionally, variables related to demographics (age at the time of surgery, gender), indications for surgery, baseline preoperative clinical/laboratory data (body mass index (BMI), the American Society of Anesthesiologists (ASA) score [17], haemoglobin, creatinine and albumin serum level), and intraoperative parameters (type and length of surgery, estimated blood loss, lowest intraoperative heart rate and lowest intraoperative mean arterial pressure) were also recorded.

\section{Frailty definition and its model}

The cumulative deficit model of frailty was used. The equally weighted deficits, as a measure of accumulated vulnerability, were recorded in the database. The ADL/IADL and the BOMC/CDT were considered abnormal if one of the assessment tools showed literature-based impairment. Deficits in two or more Geriatric Assessment (GA) domains indicated an increased risk of postoperative complications, disability or death; this was used as the cut-off score for the GA set and also as our frailty definition.

\section{The Surgical Apgar Score}

The SAS was calculated at the end of surgery from anaesthesiology records, including estimated blood loss (EBL), lowest intraoperative heart rate $(\mathrm{HR})$ and lowest intraoperative mean arterial pressure (MAP). As shown in Table I, the score is the sum of the points from each category.

\section{Outcome measure}

The primary end points were analyses of the occurrence of complications within 30 days, death 
Table I. Surgical Apgar Score (based on [4])

\begin{tabular}{|lccccc|}
\hline Test & 0 points & 1 point & 2 points & 3 points & 4 points \\
\hline Estimated blood loss $[\mathrm{ml}]$ & $>1000$ & $601-1000$ & $101-600$ & $\leq 100$ & \\
\hline Mean arterial pressure $[\mathrm{mm} \mathrm{Hg}]$ & $<40$ & $40-54$ & $55-69$ & $\geq 70$ & $\leq 55^{*}$ \\
\hline Lowest heart rate [beats/min] & $>85$ & $76-85$ & $66-75$ & $56-65$ & \\
\hline
\end{tabular}

*The occurrence of pathologic bradyarrhythmia such as sinus arrest, atrioventricular block or dissociation, junctional or ventricular escape rhythms, and asystole was equal to 0 points for the lowest heart rate.

within 30 days and 12 months follow-up after surgery. Complications were defined as any event occurring within 30 days of surgery that requires treatment not routinely applied in the post-operative period. The predefined complications were documented prospectively, allowing the complete accrual of data. The severity of complications was classified according to the Clavien-Dindo scale [18]. In the case of a complications requiring many treatment methods, the highest severity grade was recorded. Post-operative mortality was defined as death within 30 days after surgery. Additionally, the mortality was reassessed at 90, 180 days and 1 year. The follow-up consisted of an outpatient visit and a phone encounter.

\section{Statistical analysis}

The data were analysed using Statistica 12.0 software (Dell). There were no missing data. Categorical variables were described as numbers (percentages), while continuous variables were reported as median and range. Pearson's $\chi^{2}$ or Fisher's exact test was used to compare categorical variables where appropriate. The Shapiro-Wilk and the Kolmogorov-Smirnov tests, with the Lilliefors correction, were used to confirm the normality of the distribution of the continuous variables and the unpaired Student's $t$ test or the nonparametric Mann-Whitney $U$ test was used for comparisons where appropriate.

A univariate analysis was conducted investigating the association between various pre- and intraoperative operative variables with 30-day postoperative morbidity, 30-day and 12-month postoperative mortality. A multivariate analysis was conducted to identify which of the mentioned factors best described the occurrence of postoperative outcomes. The entry criteria for the model were set at $p=0.1$ in the univariate analysis and the forward stepwise logistic regression was used for those factors. Collinearity among variables was detected by means of the Spearman correlation test. Receiver operating characteristics (ROC) and the area under the curve (AUC) were used to evaluate the diagnostic performance and predictive ability of the screening tests to detect postoperative outcomes. A statistical comparison of different tests was carried out as described by Hanley and Hajin-Tilaki [19]. Statistical significance was defined as a two-sided $p \leq 0.05$.

\section{Results}

\section{Study protocol}

In the inclusion period, 161 patients were scheduled for elective laparoscopic cholecystectomy due to symptomatic gallbladder stones or polyps. Fifteen patients were excluded preoperatively: 1 patient refused to participate in the study and 14 were scheduled for an open cholecystectomy. Three patients were excluded in the postoperative period due to gallbladder cancer diagnosis in the final pathological report. A total of 144 of these patients were included in the study, among whom 142 were available for analyses at 12 months. Two patients were lost to follow-up; they did not answer any phone calls or email (Figure 1).

Consultants or residents under the direct supervision of a consultant (who also served as the first assistant) performed all the operations. Laparoscopic cholecystectomy was performed using a standard three- or four-port technique.

\section{Baseline characteristics}

The study sample comprised 144 consecutive patients (95 female (66\%), 49 male) with a median age of 76 (range: 70-91). Eighty-nine (61.8\%) patients were at the age 70-79 years, 52 (36.1\%) were 80-89 years and $3(2.1 \%)$ were 90 years old or older. Fiftyone (35.4\%) patients were assessed as having an ASA score $>2$ (Table II). 
The most common indications for surgery were: symptomatic gallbladder stones (105 patients), which comprised over $72.9 \%$ of the cases, followed by a gallbladder polyp (39 patients; $26.1 \%$ ).

\section{Descriptive analysis of GA components}

The detailed results of GA instruments are listed in Table III. Each of the validated assessment tools is presented with literature-based cut-off scores, together with the proportion of patients who had abnormal results in the test.

Sixteen patients were recognised as functionally dependent using ADL and I-ADL. Another 10\% had an abnormal time when performing the TUG. Almost $19 \%$ of the study population had some degree of malnutrition at the hospital admission. Only 8 (5.5\%) patients had no comorbidity and $21(14.6 \%)$ had one concomitant disease. Using the Geriatric Depression Scale, 20 (13.9\%) patients met the criteria for depression. Prior to the GA, only 9 patients

Table II. Baseline characteristics of study population

\begin{tabular}{|c|c|c|}
\hline \multirow{15}{*}{$\begin{array}{l}\text { Demographic } \\
\text { data }\end{array}$} & Gender: female/male & $95(66 \%) / 49(34 \%)$ \\
\hline & \multicolumn{2}{|l|}{ Age, $n(\%)$ [years]: } \\
\hline & $70-79$ & $89(61.8)$ \\
\hline & $80-89$ & $52(36.1)$ \\
\hline & $\geq 90$ & $3(2.1)$ \\
\hline & Median age [years] (range) & $76(70-91)$ \\
\hline & \multicolumn{2}{|l|}{ BMI, $n(\%)\left[\mathrm{kg} / \mathrm{m}^{2}\right]$ : } \\
\hline & $<20$ & $6(4.2)$ \\
\hline & $20-24.9$ & $30(20.8)$ \\
\hline & $25-29.9$ & $73(50.7)$ \\
\hline & $\geq 30$ & $35(24.3)$ \\
\hline & \multicolumn{2}{|l|}{ ASA score, $n(\%)$ : } \\
\hline & Score 1 & $10(6.9)$ \\
\hline & Score 2 & $83(42.3)$ \\
\hline & Score 3 & $51(35.4)$ \\
\hline \multirow{4}{*}{$\begin{array}{l}\text { Preoperative } \\
\text { laboratory } \\
\text { results }\end{array}$} & Median (range): & \\
\hline & Haemoglobin [g/dl] & $13.6(8.2-17.1)$ \\
\hline & Creatinine $[\mu \mathrm{mol} / \mathrm{l}]$ & $78.5(48-154)$ \\
\hline & Albumin $[\mathrm{g} / \mathrm{l}]$ & $44(31-56)$ \\
\hline \multirow{3}{*}{$\begin{array}{l}\text { Final } \\
\text { gallbladder } \\
\text { pathology } \\
\text { report }\end{array}$} & Gallbladder stones & $114(79.2 \%)$ \\
\hline & Chronic inflammation & $21(14.6 \%)$ \\
\hline & Benign neoplastic polyps & $9(6.3 \%)$ \\
\hline
\end{tabular}

ASA - American Society of Anesthesiologists (ASA), ${ }^{*}$ retroperitoneal liposarcoma. had such a diagnosis. An even bigger difference was observed in the case of cognitive tests. The BOMC/ CDT was impaired for a total of $23.6 \%$ of patients. Prior to their submission, this was known in only $10(6.9 \%)$ patients. The median number of drugs taken by the patient was 4 (range: $0-16$ ).

\section{Frailty frequency}

The prevalence of frailty was $44.4 \%$ ( 64 patients), as determined by the cumulative deficit model with
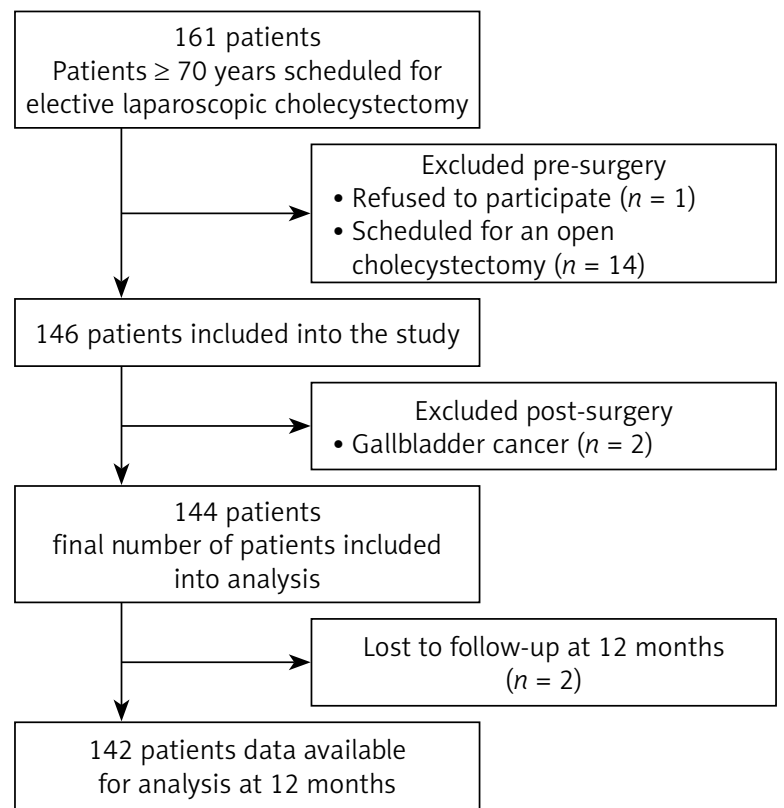

Figure 1. Study recruitment and follow-up

Table III. Abnormal geriatric assessment

\begin{tabular}{|lc|}
\hline Test & Patients, $n$ (\%) \\
\hline ADL (cut-off score $<$ 5) & $10(6.9)$ \\
\hline IADL (cut-off score $\leq 7$ ) & $23(16)$ \\
\hline MNA full (cut-off score $<$ 24) & $27(18.8)$ \\
\hline CCS (cut-off score $\geq 3$ ) & $19(13.2)$ \\
\hline TUG (cut-off score $\geq 20$ s) & $14(9.7)$ \\
\hline GDS (cut-off score $>$ 5) & $20(13.9)$ \\
\hline BOMC test (cut-off score $>10)$ & $14(9.7)$ \\
\hline CDT-test (cut-off score $>3)$ & $34(23.6)$ \\
\hline Polypharmacy $($ cut-off score $>7)$ & $41(28.5)$ \\
\hline MOS-SSS (cut-off score $<4$ ) & $13(9.0)$ \\
\hline
\end{tabular}

$A D L$ - activities of daily living, $I A D L$ - instrumental activities of daily living, $B O M C$ - Blessed Orientation-Memory-Concentration Test, CDT-test - Clock Drawing Test, CCS - Charlson Comorbidity Scale, GDS - Geriatric Depression Scale, TUG - Timed Up and Go, MNA - full nutritional assessment, MOS-SSS - Medical Outcomes Study Social Support Scale. 
Table IV. Summary of 30-day postoperative morbidity according to the Clavien-Dindo classification

\begin{tabular}{|c|c|c|c|c|c|c|}
\hline $\begin{array}{c}\text { C-D } \\
\text { Grade } 1\end{array}$ & $\begin{array}{c}\text { C-D } \\
\text { Grade } 2\end{array}$ & $\begin{array}{c}\text { C-D } \\
\text { Grade 3a }\end{array}$ & $\begin{array}{c}\text { C-D } \\
\text { Grade } 3 b\end{array}$ & $\begin{array}{c}\text { C-D } \\
\text { Grade 4a }\end{array}$ & $\begin{array}{c}\text { C-D } \\
\text { Grade } 4 b\end{array}$ & $\begin{array}{c}\text { C-D } \\
\text { Grade } 5\end{array}$ \\
\hline $\begin{array}{c}\text { Renal } \\
\text { insufficiency } 1\end{array}$ & Pneumonia 3 & $\begin{array}{l}\text { Abdominal } \\
\text { abscess } 1\end{array}$ & $\begin{array}{l}\text { Abdominal } \\
\text { abscesses } 1\end{array}$ & $\begin{array}{c}\text { Circulatory } \\
\text { insufficiency } 1\end{array}$ & & \\
\hline $\begin{array}{l}\text { Wound } \\
\text { infection } 1\end{array}$ & $\begin{array}{l}\text { Wound } \\
\text { infection } 1\end{array}$ & $\begin{array}{c}\text { Wound } \\
\text { dehiscence } 1\end{array}$ & Biliary fistula 2 & $\begin{array}{c}\text { Respiratory } \\
\text { insufficiency } 1\end{array}$ & & \\
\hline & \multirow[t]{2}{*}{$\begin{array}{c}\text { Atrial } \\
\text { fibrillation } 2\end{array}$} & & $\begin{array}{l}\text { Postoperative } \\
\text { bleeding } 1\end{array}$ & & & \\
\hline & & & $\begin{array}{c}\text { Intestinal } \\
\text { perforation } 1\end{array}$ & & & \\
\hline 2 & 7 & 2 & 5 & 2 & 0 & 0 \\
\hline
\end{tabular}

cut-off at the level of two or more abnormal GA domains. There was no statistically significant difference between the number of frail female and male patients: 39 (41\%) vs. 25 (51\%); $p>0.05$

\section{Early outcome}

The 30-day mortality was $0 \%$. Thirty-day morbidity occurred in 17 (11.8\%) patients, including $9(6.3 \%)$ patients with major complications, grade 3-4 according to the Clavien-Dindo classification (Table IV). Conversion was necessary in 12 (8.3\%) patients, mostly due to chronic inflammation, gallbladder-colon fistula, and 1 case of intraoperative bleeding. Five patients had to be reoperated on due to postoperative complications (3.5\%). The median length of postoperative stay was 2 (1-38) days.

\section{Follow-up outcome}

Of the 142 patients available for analysis after 12 months, 9 (6.3\%) patients died, 127 (89.4\%) lived

Table V. Results of bivariate $\chi^{2}$ test between the SAS and 30-day major mortality in fit and frail older patients

\begin{tabular}{|lccc|}
\hline SAS [points] & $\begin{array}{c}\text { FIT } \\
\text { 30-day morbidity } \\
n(\%)\end{array}$ & $\begin{array}{c}\text { FRAIL } \\
\text { 30y morbidity } \\
n(\%)\end{array}$ & $P$-value \\
\hline $7-10$ & $2(3)$ & $4(8)$ & 0.2 \\
\hline$<7$ & $6(50)$ & $5(35.7)$ & 0.5 \\
\hline SAS [points] & 30-day morbidity, $n(\%)$ & $P$-value \\
\hline $7-10$ & \multicolumn{3}{c}{$6(5.4)$} \\
\hline$<7$ & $11(42.3)$ & $<0.001$ \\
\hline 5 SAS - Surgical Apgar Score.
\end{tabular}

in their own home, and 6 (4.3\%) lived in a nursing home. Seven deaths occurred in the frail patients ( 3 in the first 3 months, another 2 between the $3^{\text {rd }}$ and $6^{\text {th }}$ month and 2 between the $6^{\text {th }}$ and $12^{\text {th }}$ postoperative months). Two deaths occurred in the fit patients, in the $4^{\text {th }}$ and $7^{\text {th }}$ postoperative month.

\section{The SAS score}

Analysing the intraoperative variables, the median lowest heart rate was 50 (range: 25-90) beats/ min, the median lowest arterial pressure was 60 (range: $36-98) \mathrm{mm} \mathrm{Hg}$ and the median estimated blood loss was 50 (range: 0-850) ml. The median operative duration was 79 (range: 30-190) min. None of the above variables alone was identified as a risk factor of 30-day morbidity in the regression analysis.

Table $V$ presents the results of bivariate $\chi^{2}$ tests between the SAS score and postoperative 30-day morbidity among fit and frail patients. Patients scoring 7-10 and $<7$ points had 30-day morbidity rates of $3 \%$ vs. $8 \%(p=0.2)$, and $50 \%$ and $35.7 \%(p<0.5)$ in fit and frail older patients, respectively.

The lower part of Table $V$ shows 30-day combined morbidity. The older patients with the SAS $<7$ had significantly more complications than those with the SAS of 7 or more points. The difference was even more significant if surgical complications (biliary fistula, postoperative bleeding, abdominal abscess) were excluded from the comparison.

In the case of 1-year mortality, the difference between the fit and the frail patients was significant ( $p<0.05) ; 7$ out of 9 deaths occurred in the frail group. Three patient with the SAS score $<7$ points $(18.8 \%)$ and $6(5.1 \%)$ patients with the SAS $\geq 7$ points died in the follow-up (Table VI). 


\section{Risk factors of 30-day postoperative outcome}

The SAS turned out to be a predictive factor of 30-day postoperative morbidity in a univariate regression analysis. In turn, age $\geq 85$ and frailty were predictors of 1-year mortality. The detailed results of univariate logistic regression are shown in Table VII.

Multivariate analyses (adjusted by age, gender, $B M I$, albumin serum level and length of surgery, frailty, ASA > 2) have identified only the SAS score as an independent factor that predicts 30-day postoperative complications $(\mathrm{OR}=5.1 ; 95 \% \mathrm{Cl}: 1.5-18.1)$. In the case of 1-year mortality only age $\geq 85$ years $(\mathrm{OR}=1.9 ; 95 \% \mathrm{Cl}: 1.2-16.4)$ and frailty $(3.4 ; 95 \% \mathrm{Cl}$ : 1.1-19.3) were independent predictors. The SAS turned to be an insignificant predictive factor of 1 -year mortality $(p<0.15)$.

\section{Diagnostic accuracy}

Table VIII summarizes the sensitivity, specificity, positive predictive value (PPV), negative predictive value (NPV) and the AUC, in the ROC plot, of the SAS $<7$ points. In the case of 30-day morbidity, the AUC of the SAS was 0.71 , suggesting that it has reasonable discrimination. The other statistics such as
Table VI. Results of bivariate $\chi^{2}$ test between the SAS and 1-year mortality in fit and frail older patients

\begin{tabular}{|lccc|}
\hline SAS [points] & $\begin{array}{c}\text { FIT } \\
\text { 1-year mortality } \\
n(\%)\end{array}$ & $\begin{array}{c}\text { FRAIL } \\
\text { 1-year mortality } \\
n(\%)\end{array}$ & $P$-value \\
\hline $7-10$ & $1(1.47)$ & $5(10)$ & 0.03 \\
\hline$<7$ & $1(8.3)$ & $2(14.3)$ & 0.6 \\
\hline
\end{tabular}

SAS - Surgical Apgar Score.

sensitivity, specificity, PPV and NPV were $50 \%, 84 \%$, $29 \%$ and $93 \%$, respectively.

\section{Calibration of prediction scores}

The fit of the model was good in all cases, as shown by the Hosmer and Lemeshow test $(p>0.05)$.

\section{Discussion}

The present study demonstrates that elective laparoscopic cholecystectomy can be safely performed in older fit and frail patients with no 30-day mortality and acceptable short postoperative morbidity. There was no difference in morbidity between the fit and the frail older patients. The SAS was the only

Table VII. Results of univariate logistic regression of different pre- and intraoperative variables on the postoperative 30-day major morbidity, 30-day mortality and 1-year mortality

\begin{tabular}{|lcccc|}
\hline Pre-/intraoperative variables & \multicolumn{2}{c}{ 30-day major morbidity } & \multicolumn{2}{c|}{ 1-year mortality } \\
\cline { 2 - 5 } & OR & $95 \% \mathrm{Cl}$ & OR & $95 \% \mathrm{Cl}$ \\
\hline Age [years] & 0.99 & $0.9-1.08$ & 0.9 & $0.8-1.1$ \\
\hline Age $\geq 85$ & 1.2 & $0.4-3.3$ & $\mathbf{2 . 3}$ & $\mathbf{1 . 3}-\mathbf{1 8 . 2}$ \\
\hline Gender (female) & 0.7 & $0.3-2.0$ & 0.8 & $0.2-2.8$ \\
\hline BMI [kg/m²] & 0.9 & $0.8-1.1$ & 0.9 & $0.8-1.1$ \\
\hline ASA $\geq 2$ & 0.9 & $0.4-2.86$ & 0.8 & $0.5-3.2$ \\
\hline Length of surgery [min] & 1.01 & $0.998-1.017$ & 1.01 & $0.99-1.02$ \\
\hline Frailty & 1.4 & $0.5-4.1$ & $\mathbf{5 . 6}$ & $\mathbf{1 . 1}-\mathbf{2 7 . 2}$ \\
\hline SAS $<$ 7 points & $\mathbf{1 3 . 7}$ & $\mathbf{4 . 4 - 4 2 . 4}$ & $\mathbf{2 . 9}$ & $0.7-13.2$ \\
\hline
\end{tabular}

BMI - body mass index, SAS - Surgical Apgar Score, OR - odds ratio, 95\% CI - 95\% confidence interval, *Statistically significant values are marked in bold.

Table VIII. Summary statistics of the SAS for predicting 30-day morbidity

\begin{tabular}{|lccccc|}
\hline & $\begin{array}{c}\text { Sensitivity } \\
(95 \% \mathrm{Cl})\end{array}$ & $\begin{array}{c}\text { Specificity } \\
(95 \% \mathrm{Cl})\end{array}$ & $\begin{array}{c}\text { Positive predictive } \\
\text { value }(95 \% \mathrm{Cl})\end{array}$ & $\begin{array}{c}\text { Negative predictive } \\
\text { value }(95 \% \mathrm{Cl})\end{array}$ & AUC (SE; 95\% CI) \\
\hline SAS $<7$ & $50(41-58)$ & $84 \%(77-89)$ & $29 \%(22-37)$ & $93 \%(88-97)$ & $0.71(0.07 ; 0.6-0.8)$ \\
\hline
\end{tabular}

SAS - Surgical Apgar Score, AUC - area under the curve, Cl - confidence interval. 
independent predictor of 30-day morbidity, which makes it a potential target for further care improvement. The chronological age and the ASA score were not found to be predictive of morbidity. However, frailty and age $>85$ years were predictors of 1-year mortality.

There is no doubt that chronological age alone is a poor descriptor of preoperative health status due to the great heterogeneity in the older population [20]. Therefore, the CGA is recommended in the preoperative evaluation of older patients, identifying up to $40 \%$ of new age-related problems that were not routinely detected in a standard history and physical examination. It also allows for the risk stratification and leads to a better estimation of residual life expectancy [20]. This is also clearly visible in our study, where frailty, assessed on the basis of CGA, was the predictor of 1-year mortality. In practice, this can be used for better preoperative selection of patients in terms of a long postoperative period. At present, there are many tools to evaluate frailty, including screening instruments. However, they still need further research and the CGA is the key element of modern geriatric care.

In our case series, there was no difference in postoperative morbidity between frail and fit patients, which implies that, as opposed to open surgery, laparoscopic cholecystectomy can, to some extent, "compensate" the perioperative risk associated with frailty. Additionally, in the case of moderate risk surgical procedures, such as cholecystectomy, the injury is too small to break the homeostasis, even in frail patients. Thus, in open surgery there is a clear correlation between frailty and morbidity, including high-risk operations such as colon resection [21]. The frail patients had higher morbidity, which was significantly smaller if they were operated on laparoscopically. In our study, the conversion rate was low, which makes a detailed evaluation difficult. However, it was correlated with an increase in postoperative morbidity although in all cases (apart from one) it was a conscious decision and not forced by intraoperative complications. On the other hand, only $35 \%$ of frail patients in our case series had more than 4 impaired domains, which could be equivalent to "severe frailty". Therefore, to evaluate the influence of frailty further, it would be useful to include only patients from this subpopulation in the analysis.

It seems that the influence of the surgical injury is a critical element in laparoscopic cholecystectomy
[22]. In our case series, only the SAS turned to be an independent risk factor. Our results are consistent with those obtained by other authors and summarized in the review on the SAS by Nair et al. published in 2017 [5]. Twenty out of 25 retrospective and 9 out of 11 prospective studies confirmed that the SAS correlated with postoperative morbidity and mortality. The cutoffs were different between the studies but most of the authors used 6/7 points to differentiate between low- and high-risk patients [5]. However, to the best of our knowledge, this is the first study reporting on the SAS in frail older patients. Similarly, we observed that the SAS was a good predictor of postoperative morbidity and mortality in older patients undergoing an emergency surgery $[6,23]$. There were also reports showing that the SAS had no correlation with the outcome: in orthopaedic procedures (mainly carried out under spinal anaesthesia) [24], in the microvascular head and neck reconstruction [25], but also in hysterectomy [26], oesophagectomy [27] and gastrectomy [28].

Moreover, none of the SAS components alone turned to be significant; only a combination of them did. A further target of improvement could be the patients with the SAS $<7$. This allows identification of high risk patients who can be followed meticulously to decrease the "failure to rescue" rate as much as possible.

There are also some limitations to our study. This was a single institution analysis in which several surgeons (including residents) performed the operations. However, only consultants operated on all patients with postoperative surgical complications due to their complexity. There was also inherent variability in the value of estimated blood loss (measurements dictated by the surgeon performing the operation). Moreover, only patients referred to our outpatient clinic were included in the study, which is not an accurate picture of the older population. Despite these limitations, this is the first reported study to prospectively assess the efficacy of the SAS for older frail patients undergoing laparoscopic cholecystectomy. Another significant strength is that we used the Comprehensive Geriatric Assessment to diagnose frailty, and not screening tests.

\section{Conclusions}

Laparoscopic cholecystectomy can be safely performed in older fit and frail patients with no mortality, acceptably short postoperative morbidity and low 1-year mortality. The SAS, not age and not the ASA 
score, turned out to be the most important predictor of 30-day morbidity. This implies that the course of surgery is a critical element in the treatment of older patients undergoing laparoscopic cholecystectomy. Older patients with the SAS $<7$ points should be followed meticulously in order to diagnose and treat potential complications early on.

\section{Conflict of interest}

The authors declare no conflict of interest.

\section{References}

1. Nielsen LB, Harboe KM, Bardram L. Cholecystectomy for the elderly: no hesitation for otherwise healthy patients. Surg Endosc 2014; 28: 171-7.

2. Robinson TN, Eiseman B, Wallace J, et al. Redefining geriatric preoperative assessment using frailty, disability and co-morbidity. Ann Surg 2009; 250: 449-55.

3. Kenig J, Wałęga P, Olszewska U, et al. Geriatric assessment as a qualification element for elective and emergency cholecystectomy in older patients. World J Emerg Surg 2016; 11: 36-43.

4. Gawande AA, Kwaan MR, Regenbogen SE, et al. An Apgar score for surgery. J Am Coll Surg 2007; 204: 201-8.

5. Nair A, Bharuka A, Rayani BK. The reliability of surgical Apgar score in predicting immediate and late postoperative morbidity and mortality: a narrative review. Rambam Maimonides Med J 2018; 9: 1-8.

6. Kenig J, Mastalerz K, Lukasiewicz K, et al. The Surgical Apgar score predicts outcomes of emergency abdominal surgeries both in fit and frail older patients. Arch Gerontol Geriatr 2018; 10: 54-9.

7. Katz S, Akpom CA. A measure of primary sociobiological function. Int J Health Serv 1976; 6: 493-7.

8. Lawton MP, Brody EM. Assessment of older people: self-main taining and instrumental activities of daily living. Gerontologist 1969; 9: 179-86.

9. Tomlinson BE, Roth $M$. The association between quantitative measures of dementia and senile change in cerebral grey matter of elderly subjects. Br J Psychiat 1968; 114: 797-11.

10. Watson YI, Arfken CL, Birge SL. Clock completion: an objective screening test for dementia. J Am Geriatr Soc 1993; 41: 1235-40.

11. Brink TL, Yesavage JA, Lum O, et al. Screening test for geriatric depression. Clin Gerontol 1982; 1: 37-44.

12. Charlson ME, Pompei P, Ales KL, MacKenzie CR. A new method of classifying prognostic comorbidity in longitudinal studies: development and validation. J Chronic Dis 1987; 40: 373-83.

13. Guigoz Y, Vellas B, Garry PJ. Mini Nutritional Assessment: a practical assessment tool for grading the nutritional state of elderly patients. Facts Res Gerontol 1994; 4 (Suppl 2): 15-60.

14. Curtis LH1, Østbye T, Sendersky V, et al. Inappropriate prescribing for elderly Americans in a large outpatient population. Arch Intern Med 2004; 164: 1621-5.

15. Podsiadlo D, Richardson S. The Timed “Up \& Go": a test of basic functional mobility for frail elderly persons. J Am Geriatr Soc 1991: 39: 142-8.
16. Sherbourne CD, Stewart AL. The MOS Social Support Survey. Soc Sci Med 1991; 32: 705-14

17. American Society of Anesthesiologists. New classification of physical status. Anesthesiology 1963; 24: 111.

18. Dindo D, Demartines N, Clavien PA. Classification of surgical complications. A new proposal with evaluation in a cohort of 6336 patients and results of a survey. Ann Surg 2004; 240: 205-13.

19. Hanley JA, Hajin-Tilaki KO. Sampling variability of nonparametric estimates of the areas under receiver operating characteristics curves: an update. Acad Radiol 1997; 4: 49-58.

20. Wildiers G, Heeren P, Puts M, et al. International Society of Geriatric Oncology Consensus on geriatric assessment in older patients with cancer. J Clin Oncology 2014; 32: 2595-11.

21. Mosquera C, Spaniolas K, Fitzgerald TL. Impact of frailty on approach to colonic resection. Laparoscopic vs open surgery. World J Gastroenterol 2016; 22: 9544-53.

22. Michalik M, Dowgiałło-Wnukiewicz N, Lech P, Zacharz K. Surgery of the elderly in emergency room mode. Is there a place for laparoscopy? Videosurgery Miniinv 2017; 12: 115-9.

23. Strøyer S, Mantoni T, Svendsen LB. Evaluation of the Surgical Apgar Score in patients undergoing Ivor-Lewis esophagectomy. J Surg Oncol 2017; 115: 186-91.

24. Wuerz TH, Regenbogen SE, Ehrenfeld JM, et al. The Surgical Apgar Score in hip and knee arthroplasty. Clin Orthop Relat Res 2011; 469: 1119-26.

25. Lau D, Yee TJ, La Marca F, et al. Utility of the Surgical Apgar Score for patients who undergo surgery for spinal metastasis. Clin Spine Surg 2016; 30: 374-81.

26. Ettinger KS, Moore EJ, Lohse CM, et al. Application of the Surgical Apgar Score to microvascular head and neck reconstruction. J Oral Maxillofac Surg 2017; 74: 1668-77.

27. Clark RM, Lee MS, Alejandro Rauh-Hain J, et al. Surgical Apgar Score and prediction of morbidity in women undergoing hysterectomy for malignancy. Gynecol Oncol 2015; 136: 516-20.

28. Miki Y, Tokunaga M, Tanizawa Y, et al. Perioperative risk assessment for gastrectomy by Surgical Apgar Score. Ann Surg Oncol 2014; 21: 2601-7.

Received: 21.02.2018, accepted: 14.04.2018. 